DOI: http://dx.doi.org/10.12957/demetra.2015.14699

\title{
A circulação de pessoas influencia a disponibilidade de restaurantes, bares e lanchonetes? Um estudo no município de São Paulo
}

\section{Does the circulation of people influence the availability of restaurants, bars and snack bars? Findings from the city of Sao Paulo}

\author{
Daniela Silva Canella \\ Ana Clara da Fonseca Leitão Duran' \\ Thamiris Ferreira Tavares \\ Patrícia Constante Jaime ${ }^{2}$ \\ 1 Universidade de São Paulo, Programa de \\ Pós-Graduação em Nutrição em Saúde Pública, \\ Faculdade de Saúde Pública. São Paulo-SP, Brasil. \\ 2 Universidade de São Paulo, Departamento \\ de Nutrição, Faculdade de Saúde Pública. São \\ Paulo-SP, Brasil. \\ Correspondência / Corrrespondence \\ Daniela Silva Canella \\ E-mail:dcanella@usp.br
}

\section{Resumo}

Objetivo: Descrever a distribuição de restaurantes, bares e lanchonetes no município de São Paulo; e avaliar a correlação entre a densidade desses estabelecimentos e a rede de transporte público, utilizada como proxy da circulação de pessoas. Metodologia: Estudo transversal no qual foram auditados todos os restaurantes, bares e lanchonetes e similares, encontrados nos 52 setores censitários avaliados, pertencentes a 13 diferentes distritos administrativos do município de São Paulo. Foi realizada análise descritiva dos dados e avaliada a correlação entre a densidade de estabelecimentos e da rede de transporte público, ajustada pela renda familiar no distrito administrativo onde cada estabelecimento estava localizado. Resultados e Discussão: Foram avaliados 472 restaurantes, bares e lanchonetes, sendo a maioria encontrada em áreas de nível intermediário de renda. A densidade total de estabelecimentos foi correlacionada de forma positiva com a densidade de rede de transporte público, ajustada pela renda familiar. Resultado semelhante foi encontrado quando apenas os restaurantes de comida rápida foram incluídos na análise. Em outros países, a relação entre transporte público e ambiente alimentar tem sido explorada apenas em relação ao acesso a supermercados e hipermercados e ao consequente melhor acesso a alimentos saudáveis. Conclusão: Verificou-se maior densidade de restaurantes, bares e lanchonetes em áreas com maior cobertura da rede de transporte público, assim intervenções focadas no ambiente alimentar devem priorizar áreas com maior densidade de transporte público, a fim de atingir um grande número de pessoas e estabelecimentos, garantindo a aplicabilidade dos recursos. 
Palavras-chave: Nutrição em Saúde Pública. Alimentação. Comida Rápida. Ambiente. Restaurantes. Meios de Transporte. Fatores Socioeconômicos.

\section{Abstract}

Objective: To describe the distribution of restaurants, bars and snack bars in the city of São Paulo-SP, Brazil; and to evaluate the correlation between the density of these establishments and the public transport network, used as a proxy of circulation of people. Methods: Cross-sectional study in which were audited all restaurants, bars and snack bars and the like, found in 52 census tracts assessed, belonging to 13 different administrative districts of São Paulo. Descriptive analysis was performed on data and correlation between the density of establishments and public transportation network, adjusted for family income in the administrative district where each establishment was located. Results and Discussion: A total of 472 restaurants, bars and snack bars, mostly found in intermediate income areas. The total density of establishments was positively correlated with the public transport network density, adjusted for family income. Similar results were found when only snack bars were included in the analysis. In other countries, the relationship between public transport and food environment has been explored only in relation to access to supermarkets and hypermarkets and the resulting improved access to healthy foods. Conclusion: A higher density of restaurants, bars and coffee shops in areas with greater coverage of the public transport network and focused interventions in the food environment should prioritize areas with the highest density of public transport in order to reach a large number of people and properties, ensuring the applicability of resources.

Key words: Public Health Nutrition. Food. Fast Foods. Environment. Restaurants. Transportation. Socioeconomic Factors.

\section{Introdução}

A realização de refeições fora do domicílio é cada vez mais frequente., ${ }^{1,2}$ De acordo com dados sobre o consumo alimentar dos brasileiros, $40 \%$ dos indivíduos relataram realizar refeições fora do domicílio, sendo pizza, refrigerantes, sanduíches e salgadinhos fritos e assados, alimentos característicos de restaurantes e lanchonetes de comida rápida (fast food), os mais consumidos. ${ }^{3}$ Renda elevada e proximidade às capitais dos estados estão associadas a maiores gastos com alimentação fora do domicílio. ${ }^{2}$ 
Evidências apontam que, em alguns contextos de alimentação fora do domicílio, como restaurantes de comida rápida, a qualidade nutricional da refeição é ruim, contendo altas quantidades de açúcares e óleos e gorduras, ${ }^{4-6}$ e pode estar associada ao ganho de peso e a outros desfechos em saúde. ${ }^{7,8}$ Ademais, há relação direta entre o acesso a restaurantes de comida rápida e a frequência na qual as pessoas comem nesses tipos de estabelecimentos. ${ }^{9}$

Nos últimos anos, estudos nacionais e internacionais vêm explorando o impacto da disponibilidade de alimentos não apenas ao redor do domicílio, mas também, ainda que em menor grau, ao redor do local de trabalho ${ }^{10}$ e do caminho realizado entre o domicílio e trabalho. ${ }^{11}$ Desta maneira, as características apenas do ambiente alimentar do domicílio podem não refletir de forma adequada todos os alimentos aos quais as pessoas estão expostas, já que parte importante do consumo alimentar ocorre no local de trabalho ou estudo, ou mesmo no caminho realizado entre o domicílio e trabalho/estudo. ${ }^{11}$ Já existem evidências, por exemplo, da relação entre a disponibilidade de transporte público e o acesso a supermercados e hipermercados. ${ }^{12,13}$ Assim, faz-se necessário explorar outros componentes do ambiente que possam influenciar a disponibilidade de restaurantes, bares e lanchonetes e, consequentemente, o consumo alimentar fora do domicilio, como rede de transporte público, uma proxy importante da circulação de pessoas no local.

Diante da prática crescente da alimentação fora do domicílio no Brasil, ${ }^{2}$ são escassos os estudos realizados em grandes centros urbanos que caracterizem a distribuição dos diversos estabelecimentos que comercializam alimentos e sua relação com a rede de transporte público local. Assim, o objetivo deste estudo foi descrever a distribuição de restaurantes, bares e lanchonetes no município de São Paulo e avaliar a correlação entre a densidade desses estabelecimentos e a densidade da rede de transporte público das áreas estudadas.

\section{Metodologia}

Estudo transversal, parte do Estudo do Ambiente Obesogênico (ESAO), conduzido no município de São Paulo entre novembro de 2010 e fevereiro de 2011. Por meio de coleta de dados in loco, foram registrados e avaliados todos os restaurantes, bares e lanchonetes encontrados por avaliadores treinados localizados em 52 setores censitários aleatoriamente selecionados e igualmente distribuídos em 13 distritos administrativos. Os distritos foram previamente selecionados considerando a pluralidade de relações entre as características ambientais e individuais dentre os 96 distritos administrativos existentes no município de São Paulo.

Para a amostragem dos 13 distritos administrativos incluídos no estudo, foram utilizadas medidas socioeconômicas (Índice de Desenvolvimento Humano do Município - IDH-M - desenvolvido a partir de dados sobre longevidade, educação e renda, obtidos no Censo de 2000 para cada distrito administrativo da capital paulista ${ }^{14} \mathrm{e}$ indicadores do 
ambiente alimentar urbano previamente associados à prevalência de obesidade e ao consumo alimentar em estudos internacionais. ${ }^{15,16}$ São estes: densidade de hipermercados, supermercados, pequenos mercados e feiras livres, coletados a partir de bases de dados secundários disponibilizadas publicamente pela Secretaria de Desenvolvimento Urbano do município de São Paulo: ${ }^{17}$ e densidade de restaurantes de comida rápida de quatro grandes redes nacionais e internacionais (fast food), somada às informações sobre a localização dos shopping centers da capital paulista, utilizada como proxy do número de praças de alimentação. Estas concentram, como se sabe, um grande número de restaurantes de comida rápida de grandes redes nacionais e internacionais. Os valores de densidade foram utilizados a fim de controlar o número de estabelecimentos pela população residente em cada distrito administrativo, utilizando a população segundo o Censo de 2010.18

Os restaurantes, bares e lanchonetes encontrados e avaliados foram classificados em: restaurantes à la carte, por quilo, self service, bares, padarias, cafés, sorveterias, lojas de conveniência, restaurantes de comida rápida ( fast food) de pequenas e grandes redes e outros. Para o presente estudo, considerou-se a totalidade desses estabelecimentos agrupados, chamados aqui de restaurantes, bares e lanchonetes, e os restaurantes de comida rápida (de pequenas e grandes redes nacionais e internacionais).

As variáveis estudadas foram a densidade de restaurantes, bares e lanchonetes $(\mathrm{n} / 10,000$ habitantes do distrito administrativo) e a densidade de transporte público (n/10,000 habitantes do distrito administrativo), calculada utilizando o números de estações de metrô, trem e terminais urbanos de ônibus do município de São Paulo existentes em todo o distrito administrativo em 2011. ${ }^{17,19,20}$ Para o cálculo das densidades de ambas as variáveis de estudo, utilizou-se o número de residentes de cada distrito administrativo segundo o Censo de 2010.18

Devido à distribuição não paramétrica da densidade de restaurantes, bares e lanchonetes e da rede de transporte público, o teste de Kruskal-Wallis foi utilizado para verificar a distribuição da densidade de restaurantes, bares e lanchonetes agrupados, dos restaurantes de comida rápida separadamente e da rede de transporte público, segundo terços de renda familiar do distrito administrativo. Para descrever a renda, utilizou-se a porcentagem de domicílios com baixa renda familiar, entre meio e um salário mínimo, em cada um dos 13 distritos administrativos, segundo dados do Censo de 2010.18

Posteriormente, as correlações entre as densidades da rede de transporte público e de restaurantes, bares e lanchonetes agrupados e de restaurantes de comida rápida foram avaliadas utilizando o coeficiente de correlação de Spearman. A correlação ajustada pela renda do distrito administrativo foi avaliada utilizando-se o coeficiente de correlação parcial.

Os questionários de avaliação dos estabelecimentos foram duplamente digitados, e os dados foram analisados no software Statistical Package for Social Sciences 17.0 (SPSS Inc., Chicago, IL, USA). 
Este estudo não envolveu seres humanos, mas, ainda assim, foi apresentado ao Comitê de Ética em Pesquisa da Faculdade de Saúde Pública da Universidade de São Paulo em 2009 (protocolo no 2.034).

\section{Resultados}

Foram encontrados e avaliados 472 restaurantes, bares e lanchonetes, distribuídos entre os 13 distritos auditados (média de 36 e variação de 5 a 85 estabelecimentos por distrito).

Maior densidade de restaurantes, bares e lanchonetes (mediana $=5,05$ estabelecimentos/10.000 habitantes) foi encontrada em distrito localizado em locais de renda intermediária (2o tercil). A densidade da rede de transporte público variou de 0,000 a 1,07/10.000 habitantes, não tendo sido encontrada diferença dentre os distritos nos três níveis de renda (tabela 1).

Tabela 1. Disponibilidade de restaurantes, bares e lanchonetes e rede de transporte público de acordo com a renda familiar. São Paulo-SP, 2011.

\begin{tabular}{|c|c|c|}
\hline $\begin{array}{l}\text { Terços da porcentagem de } \\
\text { famílias com renda familiar }\end{array}$ & $\begin{array}{c}\text { Densidade de restaurantes, } \\
\text { bares e lanchonetes }\end{array}$ & $\begin{array}{c}\text { Densidade de } \\
\text { Transporte Público }\end{array}$ \\
\hline $\begin{array}{c}\text { entre } 0,5 \text { e } 1,0 \text { salários } \\
\text { mínimos }^{1}\end{array}$ & $\begin{array}{c}\text { Mediana } \\
\text { (mínimo - máximo) }\end{array}$ & $\begin{array}{c}\text { Mediana } \\
\text { (mínimo - máximo) }\end{array}$ \\
\hline $1^{\mathrm{o}}$ tercil (mais ricos) & $\begin{array}{c}1,72 \\
(1,17-2,71)\end{array}$ & $\begin{array}{c}0,25 \\
(0,09-0,70)\end{array}$ \\
\hline $2^{\circ}$ tercil & $\begin{array}{c}5,05 \\
(3,14-2,62)\end{array}$ & $\begin{array}{c}0,23 \\
(0,00-0,93)\end{array}$ \\
\hline $3^{\mathrm{o}}$ tercil (mais pobres) & $\begin{array}{c}1,47 \\
(1,27-2,08)^{*}\end{array}$ & $\begin{array}{c}0,06 \\
(0,00-0,15)\end{array}$ \\
\hline
\end{tabular}

${ }^{1}$ Salário mínimo mensal brasileiro em junho de $2010=\mathrm{R} \$ 510,00$

* p $<0,05$, segundo Teste de Kruskal-Wallis

Quando analisada a correlação bruta, obtida por meio do coeficiente de correlação de Spearman, não se observou associação entre a disponibilidade de transporte público e de restaurantes, bares e lanchonetes. No entanto, verificou-se a existência de correlação forte e positiva entre a densidade de estabelecimentos e a densidade de rede de transporte público $(\mathrm{r}=0,745 ; \mathrm{p}=0,005)$, quando analisada a correlação parcial, ajustada pela porcentagem de domicílios com baixa renda 
familiar. Resultado semelhante foi encontrado para os restaurantes de comida rápida $(\mathrm{r}=0,750$; $\mathrm{p}=0,005)$, segundo a tabela 2 .

Tabela 2. Associação entre a densidade da rede de transporte público com relação à densidade de todos os tipos de restaurantes, bares e lanchonetes avaliados e à densidade de restaurantes de comida rápida (fast-food). São Paulo-SP, 2011

\begin{tabular}{lcc}
\hline \multicolumn{1}{c}{ Densidade de estabelecimentos } & \multicolumn{2}{c}{ Densidade de transporte público } \\
\cline { 2 - 3 } & $\begin{array}{c}\text { Correlação } \\
\text { brutal (r) }\end{array}$ & $\begin{array}{c}\text { Correlação } \\
\text { parcial2 (r) }\end{array}$ \\
\hline $\begin{array}{l}\text { Todos os tipos de restaurantes, bares e } \\
\text { lanchonetes }\end{array}$ & 0,047 & $0,745^{*}$ \\
Restaurantes de comida rápida (fast-food) & 0,298 & $0,750^{*}$ \\
\hline${ }^{1}$ Coeficiente de correlação de Spearman & & \\
${ }^{2}$ Coeficientes de correlação parcial, controlado pela porcentagem de domicílios com renda familiar entre meio \\
e um salário mínimo \\
${ }^{*}$ valor de p $<0,05$
\end{tabular}

\section{Discussão}

No presente estudo, observou-se maior disponibilidade de restaurantes, bares e lanchonetes em distritos administrativos de nível intermediário de renda e correlação positiva entre a disponibilidade desses estabelecimentos e maior densidade de transporte público e, consequentemente, alto fluxo de circulação de indivíduos, independentemente do nível socioeconômico do local.

Em relação à distribuição dos restaurantes, bares e lanchonetes, segundo o nível socioeconômico, resultados semelhantes ao nosso foram encontrados em Los Angeles, Califórnia, EUA, com maior densidade de restaurantes de comida rápida em áreas de renda média. O mesmo, ainda que em menor magnitude, foi observado para outros tipos de restaurante. ${ }^{21}$ Por outro lado, também nos Estados Unidos, nos estados de Mississipi, Carolina do Norte, Maryland e Minnesota, verificou-se que os restaurantes com serviço completo estavam distribuídos de forma equânime entre os bairros com diferentes níveis socioeconômicos, mas os restaurantes de comida rápida estavam mais concentrados em locais de baixo e médio nível socioeconômico. ${ }^{22}$

Em países em desenvolvimento, sobretudo aqueles que passaram por rápidas e recentes transições epidemiológica e nutricional, poucas evidências estão disponíveis na 
literatura sobre a distribuição espacial de restaurantes e sua associação com variáveis socioeconômicas ou desfechos em saúde. Em estudo realizado com dados secundários, referentes às 31 subprefeituras do município de São Paulo, encontrou-se maior densidade de lanchonetes fast food de grandes redes e de transporte público nas áreas de maior nível socioeconômico. ${ }^{23}$ Já no presente estudo, no qual não foram incluídos apenas restaurantes de comida rápida de grandes redes, maior densidade de restaurantes, bares e lanchonetes foi encontrada nos distritos administrativos de renda intermediária.

A associação entre a disponibilidade de transporte público e o ambiente alimentar tem sido pouco explorada, com estudos avaliando apenas sua relação com o acesso a supermercados e hipermercados e ao consequente maior acesso a alimentos saudáveis. ${ }^{13,24}$ Não foram encontrados estudos que relacionem a densidade de transporte público com a disponibilidade de restaurantes, bares e lanchonetes. No entanto, estudo realizado nos Estados Unidos relatou que os restaurantes de comida rápida localizavam-se principalmente em áreas de maior densidade de pessoas. ${ }^{25}$

Ainda em relação ao transporte público, na Austrália, paradas de ônibus e trens foram avaliadas em relação à disponibilidade de publicidade de alimentos. Constatou-se que a publicidade nesses locais pode ter impacto significativo nas escolhas alimentares, pois as paradas de transporte público são frequentadas por um grande número de pessoas por dia. ${ }^{26}$

Nossos achados apontam a necessidade de melhor entender a relação entre o acesso a restaurantes, bares e lanchonetes e características da vizinhança ainda pouco exploradas, como a densidade de transporte público. Encontramos que, em uma grande metrópole brasileira, as áreas onde o fluxo de indivíduos é maior, como aquelas que concentram maior rede de transporte público, também concentram restaurantes, bares e lanchonetes, com destaque para os restaurantes de comida rápida.

Considerando o impacto à saúde do consumo frequente de alimentos comumente ofertados nesses estabelecimentos, como bebidas açucaradas e outros produtos ultraprocessados, ${ }^{27-29} \mathrm{faz}$-se necessário o aprofundamento de estudos que busquem melhor entender as práticas comerciais que levam tais restaurantes a se aglomerarem ao redor de estações de trem, metrô e terminais de ônibus, o que poderá contribuir com o desenvolvimento, implementação e avaliação de intervenções, políticas e programas em saúde pública. ${ }^{30,31}$

\section{Conclusão}

Conclui-se que há relação entre a densidade de restaurantes, bares e lanchonetes e áreas de alta circulação de pessoas, avaliada por meio da densidade da rede de transporte público. 
Desta forma, em um cenário de crescimento da alimentação fora do domicílio, com significativa oferta de alimentos ultraprocessados e com alta densidade energética - em vista da relação encontrada neste estudo - intervenções focadas no ambiente alimentar priorizadas em áreas com maior densidade de transporte público atingem potencialmente grande parcela da população e estabelecimentos, garantindo a aplicabilidade dos recursos investidos.

\section{Referências}

1. Kant AK, Graubard BI. Eating out in America, 1987-2000: Trends and nutritional correlates. Preventive Medicine 2004; 38(2):243-249.

2. Claro RM, Baraldi LG, Martins APB, Bandoni DH, Levy RB. Trends in spending on eating away from home in Brazil, 2002-2003 to 2008-2009. Cad. Saúde Pública 2014; 30(7):1418-1426.

3. Bezerra IN, Souza AM, Pereira RA, Sichieri R. Consumo de alimentos fora do domićlío no Brasil. Rev. Saúde Pública. 2013; 47(Suppl.1):200s-211s.

4. Orfanos P, Naska A, Trichopoulou A, Grioni S, Boer JM, van Bakel MM, et al. Eating out of home: Energy, macro- and micronutrient intakes in 10 European countries. The European Prospective Investigation into Cancer and Nutrition. Eur. J. Clin. Nutr. 2009; 63(Supl. 4):S239-S262.

5. Kirkpatrick LS, Reedy J, Kahle LL, Harris JL, Ohri-Vachaspati P, Krebs-Smith SM. Fast-food menu offerings vary in dietary quality, but are consistently poor. Public Health Nutr. 2012; 17(4):924-931.

6. Bandoni DH, Canella DS, Levy RB, Jaime PC. Eating out or in from home: analyzing the quality of meal according eating locations. Rev. Nutrição 2013; 26(6):625-632.

7. Duffey KJ, Gordon-Larsen P, Steffen LM, Jacobs DR Jr, Popkin BM. Regular consumption from fast food establishments relative to other restaurants is differentially associated with metabolic outcomes in young adults. J. Nutr. 2009; 139(11):2113-2118.

8. Bezerra IN, Curioni C, Sichieri R. Association between eating out of home and body weight. Nutr. Rev. 2012; 70(2):65-79.

9. Inagami S, Cohen DA, Brown AF, Asch SM. Body Mass Index, neighborhood fast food and restaurant concentration, and car ownership. J. Urban Health 2009; 86(5):683-695.

10. Moore K, Diez-Roux AV, Auchincloss A, Evenson KR, Kaufman J, Mujahid M, et al. Home and Work Neighborhood Environments in Relation to Body Mass Index: the Multi-Ethnic Study of Atherosclerosis (MESA). J. Epidemiol. Community Health 2013; 67(10):846-853.

11. Burgoine T, Monsivais P. Characterising food environment exposure at home, at work, and along commuting journeys using data on adults in the UK. Int. J. Behav. Nutr. Phys. Act. 2013; 10:85.

12. Burns CM, Inglis AD. Measuring food access in Melbourne: access to healthy and fast foods by car, bus, and food in an urban municipality in Melbourne. Health Place 2007; 13(4):877-885. 
13. Sheldon M, Gans KM, Tai R, George T, Lawson E, Pearlman DN. Availability, affordability, and accessibility of a healthful diet in a low-income community, Central Falls, Rhode Island, 2007-2008. Prev. Chronic Dis. 2010; 7(2): 1-7.

14. São Paulo. Prefeitura Municipal. Fundação João Pinheiro. Atlas do trabalho e desenvolvimento da cidade de São Paulo [Internet]. São Paulo: Fundação Sistema Estadual de Análise de Dados; 2007. [acesso em 14 mar. 2009]. Disponível em: http://atlasmunicipal.prefeitura.sp.gov.br/Login/Login.aspx

15. Ball K, Timperio A, Crawford D. Neighborhood socioeconomic inequalities in food access and affordability. Health Place 2009; 15(2):578-85.

16. Morland KB, Evenson KR. Obesity prevalence and the local food environment. Health Place. 2009; 15(2):491-5.

17. São Paulo. Prefeitura. Secretaria Municipal de Planejamento. Infocidade [Internet]. [acesso em 15 jan. 2011]. Disponível em: http://infocidade.prefeitura.sp.gov.br/index.php?sub $=$ notas\&cat $=17 \&$ titulo $=U$ so $\% 20$ do $\% 20$ Solo $\% 20$ Urbano\&subtit $=\% 20-\% 20$ Notas $\% 20 \mathrm{~T} \%$ E 9 cnicas

18. Instituto Brasileiro de Geografia e Estatística. Censo 2010. Rio de Janeiro: IBGE; 2011.

19. São Paulo. Governo do Estado. Companhia do Metropolitano de São Paulo. Mapa da rede metroviária de São Paulo [Internet]. [acesso em 15 jan. 2011]. Disponível em: http://www.metro.sp.gov.br/redes/ teredes.shtml

20. São Paulo Transportes S.A. Terminais urbanos [Internet]. [acesso em 15 jan. 2011]. Disponível em: http://www.sptrans.com.br/terminais/

21. Sturm R, Cohen DA. Zoning for health? The year-old ban on new fast-food. restaurants in south LA: the ordinance isn't a promising approach to attacking obesity. Health Affairs 2009; 28(6):1088-1097.

22. Morland K, Wing S, Diez-Roux A, Poole C. Neighborhood characteristics associated with the location of food stores and food service places. Am. J. Prev. Med. 2002; 22(1):23-9.

23. Jaime PC, Duran AC, Sarti FM, Lock K. Investigating environmental determinants of diet, physical activity, and overweight among adults in Sao Paulo, Brazil. J. Urban Health 2011; 88(3):567-81.

24. Larson NI, Story MT, Nelson MC. Neighborhood Environments. Disparities in Access to Healthy Foods in the U.S. Am. J. Prev. Med. 2009; 36(1):74-81.

25. James P, Arcaya MC, Parker DM, Tucker-Seeley RD, Subramanian SV. Do minority and poor neighborhoods have higher access to fast-food restaurants in the United States? Health Place 2014; 29:10-17.

26. Settle PJ, Cameron AJ, Thornton LE. Socioeconomic differences in outdoor food advertising at public transit stops across Melbourne suburbs. Aust. N. Z. J. Public Health 2014; 38(5):414-18

27. Hu FB, Malik VS. Sugar-sweetened beverages and risk of obesity and type 2 diabetes: epidemiologic evidence. Physiol. Behav. 2010; 100(1):47-54.

28. Tavares LF, Fonseca SC, Garcia Rosa ML, Yokoo EM. Relationship between ultra-processed foods and metabolic syndrome in adolescents from a Brazilian Family Doctor Program. Public Health Nutr. 2012; 15(1):82-87. 
29. Canella DS, Levy RB, Martins APB, Claro RM, Moubarac JC, Baraldi LG, et al. Ultra-processed food products and obesity in Brazilian households (2008-2009). PLoS ONE 2014; 9(3):e92752.

30. Margetts B, Warm D, Yngve A, Sjöström M. Developing an evidence-based approach to Public Health Nutrition: translating evidence into policy. Public Health Nutr. 2001; 4(6A):1393-97.

31. Mowat D. Decisões baseadas em evidências na saúde pública. Ethos Gubernamental. 2006-2007; 4:231-48.

Recebido: 26/1/2015

Aprovado: 30/1/2015 